\title{
Words or Deeds? Choosing what to know about others
}

\author{
Erte Xiao $^{\dagger \neq}$ and Cristina Bicchieri*
}

August 2009

\begin{abstract}
Social cooperation often relies on individuals' spontaneous norm obedience when there is no punishment for violation or reward for compliance. However, people do not consistently follow pro-social norms. Previous studies have suggested that an individual's tendency toward norm conformity is affected by empirical information (i.e. what others did or would do in a similar situation) as well as by normative information (i.e. what others think one ought to do). Yet little is known about whether people have an intrinsic desire to obtain norm-revealing information. In this paper, we use a dictator game to investigate whether dictators actively seek norm-revealing information and, if so, whether they prefer to get empirical or normative information. Our data show that although the majority of dictators choose to view free information before making decisions, they are equally likely to choose empirical or normative information. However, a large majority (more than $80 \%$ ) of dictators are not willing to incur even a very small cost for getting information. Our findings help to understand why norm compliance is context-dependent, and highlight the importance of making norm-revealing information salient in order to promote conformity.
\end{abstract}

Acknowledgement: We gratefully acknowledge the Goldstone Research Unit for funding that supported this research. We received valuable comments from Daniel Houser, Roberto Weber as well as attendees of the 2008 Economic Science Association meetings.

\footnotetext{
${ }^{\dagger}$ Carnegie Mellon University, Department of Social and Decision Sciences, 208 Porter Hall, Pittsburgh, PA, 15213, 412-268-6780 (office), exiao@andrew.cmu.edu.

* University of Pennsylvania, Department of Philosophy and Wharton School, 491 Cohen Hall, 249, S. 36th

Street, Philadelphia, PA 19104, 215.898.5820 (office), cb36@sas.upenn.edu.

${ }^{\ddagger}$ Correspondence to E.X.
} 


\section{Introduction}

Social cooperation often relies on individuals' spontaneous norm obedience when there is no punishment for violation or reward for compliance. Previous studies have suggested that an individual's tendency toward norm conformity is affected by what he/she believes others would do in a similar situation (i.e. empirical expectations of norm compliance) and what he/she believes others think he/she ought to do (i.e. normative expectations of norm compliance. $)^{1}$ Yet little is known about whether people have an intrinsic desire to obtain norm-revealing empirical information (i.e. what the majority does or did) and/or normative information (i.e. what the majority think one ought to do) when making decisions. If they do, we would expect individuals not just to actively look for information, but also to be prepared to pay for it. The answer to this question is important, especially for policy-makers who want to design policies aimed at influencing people's pro-social behavior. To boost norm compliance, policy makers may have to influence people's expectations via careful diffusion of norm-relevant information. In this paper, we design novel experiments to investigate individuals' demand for empirical/normative information in an environment that involves norm conformity.

To date, a large number of interesting research findings have left little doubt that social norms can be an important motivator of individual decision-making (e.g. Fehr and Fischbacher, 2004). For example, people reciprocate trust even when it is in their own self-interest not to do so (Berg et al, 1995; Cox, 2004), and cooperate in social dilemmas even when their defection would go undetected (Ledyard, 1995). On the other hand, if social norms guide decision-making, we must explain why we see variations in norm

\footnotetext{
${ }^{1}$ See Bicchieri, 2006; Bicchieri and Xiao, 2008; Bicchieri and Chavez (2009); Cason and Mui, 1998; Bardsley and Sausgruber, 2005; Krupka and Weber, 2006; Sugden, 1998 and 2004.
} 
conformity across different situations (Dawes et al, 1977). Recent research has begun to shed light on the underlying mechanism of how norms influence behavior. One possible explanation is that individuals have a conditional preference for following a norm, provided certain empirical and normative expectations are met (Bicchieri, 2006). ${ }^{2}$ This view of social norms suggests that an individual's expectations and subsequent behavior would be affected by norm-revealing information even if it is payoff-irrelevant. Previous empirical research has shown the influence of information on pro-social behavior, even when the information is payoff irrelevant (see, e.g. Bardsley and Sausgruber, 2005; Bicchieri and Xiao, 2008; Bicchieri and Chavez, 2009; Cason and Mui, 1998; Frey and Meier, 2004; Krupka and Weber, 2008).

However, one important and as yet unanswered question is whether individuals have an intrinsic desire to obtain norm-revealing information before making a decision. The social psychology literature on informational and normative influence (Deutsch and Gerard, 1955; Kelley, 1952) tells us that, when people are uncertain about how to act because the situation is ambiguous, they will try to gather information about the behavior/expectations of others similarly situated. However, this literature does not explore people's willingness to pay for such information, even if the gathering process may itself involve some cost. Thus, the fact that gathering information is often costly raises the additional question of whether people are willing to incur costs to obtain normrevealing information.

\footnotetext{
${ }^{2}$ Assuming conditional preferences for following a social norm is different from assuming a pro-social preference. The latter implies one should not be influenced by information about other people's beliefs or behavior contrary to one's preference. (see Bicchieri (2006, Ch.3) for details). The details about the differences among empirical expectation, normative expectation and second-order expectation are discussed in Bicchieri (2006, Ch. 1).
} 
It is worth noting that empirical information does not seem to play the same role as normative information in decision-making. Bicchieri and Xiao (2008) studied a dictator game in which selected information about norm compliance/transgression by previous players was provided in order to manipulate dictators' normative and empirical expectations about other dictators' beliefs/choices in the present game. We showed that when normative and empirical information (and thus the resulting expectations) are inconsistent, individuals do what they think others would do in the same situation, even when they believe that doing so would not be met with approval. This raises an important question: would individuals spontaneously seek to acquire norm-revealing information, and if so, would they differentiate empirical from normative information? Are people more interested in knowing what others do or what others think they ought to do?

The answers to the above questions can help explain why norm conformity, in many common social situations, is often context-dependent, and also have important policy implications regarding the design of policies aimed at promoting norm conformity. The reason is that different contexts might provide different information conditions that influence people's expectations and choices. Many scholars have argued for the importance of making norms salient (see Bicchieri (2006, Ch.2) for a review). Cialdini et al. (1990)'s “focus theory of norms" provides field experiment evidence that a social norm must be made salient in order to effectively influence individuals' decision-making. Making a norm salient typically means providing people with information about the behavior/beliefs of other individuals similarly situated. One implication of this theory is that even if norm-revealing information influences people's decision-making, in many 
environments in which norm-revealing information is absent or ambiguous, a majority of people might not actively seek such information when making decisions, especially if there is no cost to norm violation. That is, if most people were to actively seek normrelevant information even when it is costly, then we should not expect any significant effect from reminding people of a norm's existence. To the best of our knowledge, however, no direct evidence has been provided about individuals' willingness to acquire norm-revealing information ${ }^{3}$. If most people do not make any effort to attain normrevealing information, then institutions might need to actively disseminate such information in order to enhance norm compliance.

In this paper, we design a variant of a dictator experiment to investigate whether a large number (at least 50\%) of individuals intrinsically value norm-revealing information by offering them the possibility of acquiring empirical and normative information about past norm compliance by other parties in the same situation they are now facing. We find that although a majority (75\%) of people do not avoid norm-revealing information in decision-making, most people (more than $80 \%$ ) do not place significant value on this information. We discuss the implications of this result for understanding variations in norm compliance and the related policy implications.

\section{Experiment Design}

\footnotetext{
${ }^{3}$ Willingness to acquire norm-revealing information is different from willingness to know the final amount the receiver earns, as studied in Dana, Weber and Kuang (2007). Their paper provides experimental evidence that a significant number of dictators prefer not to reveal whether their choices hurt or help their respective receivers. The key is that, because the receivers do not know if their respective dictators know the receiver's earnings, dictators who make a selfish choice can appear to be fair both in their own eyes as well as in the eyes of their receivers. In fact, if dictators were to care enough about the receiver's payoff, we would expect dictators to have an intrinsic motivation to obtain norm-revealing information.
} 
Our experiment is based on dictator games that have been widely used to study fairness or beneficence motives. In the standard Dictator game, two subjects are paired randomly, with one acting as dictator (divider in the instructions) and the other as receiver (counterpart in the instructions). The dictator decides how much of $\$ 10$ s/he wants to send to the receiver and the receiver earns that amount. A common observation is that people make very different decisions and hold different beliefs regarding which decisions ought to be made in these games (Bardsley, 2007; Xiao \& Houser, 2008; Guala \& Mittone, 2008). ${ }^{4}$ These variations in beliefs and behavior, common in dictator games, reveal a great deal of uncertainty and confusion about what is an appropriate decision in the dictator game. Indeed, Bicchieri and Xiao (2008) provided experimental evidence that dictators' beliefs regarding what other dictators in the experiment would do or believe one ought to do can be easily influenced by what they learn about the behavior or beliefs of a majority of dictators in another single session. Thus, the ambiguity about the appropriate decision in a dictator game makes it a good tool for investigating the demand for information about norms guiding behavior in such situations.

Our experiment consists of three treatments. We first examine whether dictators want to obtain norm-relevant information at all when it is provided for free. After finding a preference for getting relevant information, we add a small cost (15 cents) to the information and test whether people value the information and whether they value the empirical information more or less than the normative information.

\footnotetext{
${ }^{4}$ In a questionnaire conducted with college students (Bicchieri, 2006), it was found that about half of the respondents (56\%) answered that no outcome in the dictator game can be said to be unfair. When explicitly asked what is a fair outcome, $68 \%$ of the subjects indicated the equal split, but $21 \%$ thought that keeping everything was fair.
} 


\section{Free Information Treatment (FI)}

In this treatment, dictators were given the option to view, at no cost, one of the two following pieces of information: (A) The offers chosen by $60 \%$ of dividers in a previous session of this experiment (i.e. empirical information); or (B) The offers that $60 \%$ of dividers in a previous session of this experiment said dividers should make (i.e. normative information). Dictators could choose not to view any information or, if choosing to view information, they had to choose either A or B, but not both. They could also choose to view this information before or after making a decision. It was made clear to the dictators that the information was available only to dictators and not to receivers.

To implement this design, we gave each dictator five sealed envelopes labeled I, II, III, IV and V. Each envelope contained a decision-making card on which the dictator could write down his/her decision, and one (or none) information card. Each envelope was labeled with instructions so that the dictator knew which envelope to open depending on his/her choices. The content of the label on each envelope is listed in Table 1. Dictators were also instructed to open only one of the envelopes. Copies of the instructions are found in Appendix A.

\section{Buy Normative Information Treatment (BN) and Buy Empirical Information Treatment (BE)}

In the "Buy Normative Information Treatment" $(\mathrm{BN})$, dictators were given an option to view the normative information. Dictators were given three envelopes and could choose one. The labels of the three envelopes are listed in Table 1. Dictators had to pay 15 cents to view the information before making a decision. The cost of 15 cents is arbitrary. To 
maximize the chance of finding out the dictators' willingness to obtain the costly information, we started with a very small amount. If dictators are not even willing to pay 15 cents for the information, this fact would suggest that the dictators will not actively seek the information when making decisions.

It is possible that the dictator is simply curious about other dividers’ past behavior and is willing to pay the cost, but not for the purpose of making a decision. In view of this possibility, we also provide the dictator the option of paying a smaller cost (5 cents) to view the information after making a decision. ${ }^{5}$

The "Buy Empirical Information” treatment (BE) is designed exactly like the BN treatment except for the fact that the information available to dictators is the offers chosen by $60 \%$ of dividers in a previous session of this experiment (i.e. empirical information). In particular, labels for the BE treatment are the same as those for the BN treatment but the content of the information card is different between the two treatments.

It is natural to ask whether the information dictators obtain affects their decisionmaking. Bicchieri and Xiao (2008) designed a version of a dictator game in which all the dictators were given either normative information or empirical information or both. They found that the information significantly affects dictators' decision-making by changing their expectations about what other dictators will do/believe should be done in the present experiment.

Since our goal here is to investigate how and if people gather norm-relevant information, we allow dictators to choose what information they want to have. As a result, self-selection is unavoidable in this experiment. That is, dictators who choose one

\footnotetext{
${ }^{5}$ It is possible that participants will perceive the price for information as signaling the value of information. If so, they would be more likely to view the information when the price is higher, and this would bias our design against our hypothesis that people will not incur costs to seek norm-relevant information.
} 
type of information might be different from those who choose another, different type of information. Moreover, the dictators who choose to get the information might be different from those who do not. Although we vary the previous sessions of dictator games we reported to the dictators as we did in Bicchieri and Xiao (2008) (see Table 2 for the content of the messages), our experiment is different from the previous one, in that it does not study the behavioral effects of information about what other people did/believed should be done. Thus, our analysis of the results will only focus on the dictators’ decisions regarding whether to acquire information.

\section{Procedure}

One hundred and fifty four students at the University of Pennsylvania participated in the study. As the information provided in this dictator game is the same as the messages used in Bicchieri and Xiao (2008), we excluded all the 254 subjects who participated in our previous experiment. Each subject received a $\$ 5$ show-up bonus in addition to the money earned in the game. Subjects were in the lab for less than one hour.

Each subject was randomly assigned the role of dictator or receiver. Dictators and receivers were separated throughout the experiment. Each subject was randomly assigned a letter as his or her ID for the duration of the experiment. A receiver and a dictator were paired if they held the same letter. All subjects received an instruction sheet explaining the rules of the game. In addition to the instructions about the dictator game, each dictator also received a separate sheet with instructions about what information was available and the cost, if any, of viewing the information, what each envelope contained, and which envelope they should open depending on their decisions. 
Copies of instructions are in Appendix A. The game started after every subject finished the instructions.

Each subject played the game exactly once. Our procedures ensured it was clear to dictators that no one, including the experimenters, knew their decisions. Each dictator was asked to open one sealed envelope and use the decision card found in the envelope to indicate their chosen split, write down their ID on the back of the decision card and then put the card and all the envelopes (including those they did not open) into a blank envelope. After all dictators had finished, the experimenter collected all the envelopes and then gave each receiver his or her dictator's envelope according to the ID. At the end of the experiment, subjects' earnings were put in envelopes marked with ID letters. Each subject picked up his/her earnings envelope privately.

\section{Results}

We obtained observations on 154 subjects: 33 pairs in the FI treatment; 24 pairs in the BE treatment; and 20 pairs in the $\mathrm{BN}$ treatment. One dictator in the FI treatment opened three envelopes. Thus, we dropped this data and kept 32 pairs in the FI treatment.

\section{Demand for free information}

Figure 1 plots the percentages of dictators who chose to view the free normative or empirical information before or after making decisions. As shown in Figure 1, the majority of dictators (75\%) chose to view the messages before making decisions, and this number is significantly greater than $50 \%(\mathrm{p}<0.01$, Binomial test $)$. However, among these dictators, no significant difference exists between the percentage of dictators who chose 
to view normative information and the percentage of dictators who chose to view empirical information ( $34.4 \%$ vs. 40.6, Z-test, $\mathrm{p}=0.61$ ). Combined with the findings in Bicchieri and Xiao (2008), this result suggests that although empirical information, and the consequent empirical expectations about what other dictators will do, dominates individuals' decision-making, especially when it contradicts normative information, people do not place different weights on empirical and normative information when they are presented separately and one has to choose one of them.

Among the 24 (of 32) dictators who viewed the free messages before making a decision, twelve were given selfish empirical or normative information and the other twelve were given fair empirical or normative information. ${ }^{6}$ Five out of the twelve who saw a message indicating fair behavior/beliefs gave the receiver at least $40 \%$. On the other hand, among the twelve who saw a message indicating selfish beliefs/behavior, only three gave the receiver $40 \%$ or more. Six dictators chose not to view the free messages either before or after their decisions; one of them chose to give the receiver $40 \%$ and the other five gave the receiver $20 \%$ or less. Two dictators chose to view the empirical information after decisions. One of them gave the receiver $40 \%$ and the other gave $10 \% .^{7}$

The fact that the majority of dictators chose to view the free message only indicates that people do not try to avoid norm-relevant information. However, it does not tell us whether individuals place any value on the information and whether they are

\footnotetext{
${ }^{6}$ Again, fair information messages were generated from the data taken from a previous session where the majority of dictators made fair offers and selfish information messages summarize the data from a previous session where the majority of dictators made selfish offers. It is clear to the dictators that the data only reveal the behaviors or the opinions of the dictators in one previous session.

${ }^{7}$ As we already mentioned, our aim was not to assess the influence of information on choices, and indeed the data we have are not sufficient to draw such conclusions. We can say, however, that we detected a strong tendency to make choices (selfish/fair) consistent with the information (selfish/fair) received.
} 
willing to pay a cost to obtain it. We next report dictators' behavior in the Buy Information treatments.

\section{Demand for Costly Information}

Although the majority of dictators in the free information treatment chose to view messages before making their decisions, most dictators chose not to view the information before making a decision when they had to pay a very small cost (15 cents). Nor were most dictators willing to pay an even smaller cost (5 cents) to view the information after making a decision. In the Buy Empirical Information treatment, only 3 out of 24 dictators, before making their decisions, paid 15 cents to find out what $60 \%$ of dictators chose in a previous session of the experiment. In the Buy Normative Information treatment, 3 out of 20 dictators paid 15 cents prior to making a decision to view what $60 \%$ of dictators in a previous session said dictators should choose. In both treatments, the percentage of dictators who paid to view the information was significantly less than $50 \%$ $(\mathrm{p}<0.01$, Binomial test). Two dictators in each treatment chose to pay 5 cents to view the information after their decisions. Figure 2 plots the distribution of dictators' choices to buy information.

Thus, our data suggest that although most people do not avoid norm-revealing information when making decisions, they seem unwilling to gather this information when there is an associated cost. Moreover, and interestingly, even though empirical information about other dictators' behavior play a more significant role in decisionmaking than information about what others think one should do, especially when the two kinds of information are jointly presented and in conflict (Bicchieri and Xiao, 2008), 
dictators do not differentiate between these two types of information when they are presented separately. Our results show that dictators are equally likely to gather both kinds of information when it is free, and neither of them when there is a small associated cost.

\section{Discussion}

Previous research has shown that social norms play an important role in individual decision-making and that norm-revealing information has a significant effect on behavior. Bicchieri and Xiao (2008) provide experimental evidence that empirical information dominates decision-making when this information is inconsistent with normative information. In this paper, we investigate whether there is a demand for normrevealing information and, if so, whether people differentiate empirical information from normative information. Our data show that although the majority of dictators choose to view the free information before making a decision, they are equally likely to choose either empirical information or normative information. Moreover, a majority of dictators are not willing to pay for the information.

It is worth noting that, in our experiment, we exclude any possible consequences of norm violation, such as punishment or negative reputation effects. It is possible that the demand for norm-relevant information will increase, for example, when there are third-party observers or even the possibility of being punished (e.g. in ultimatum game). However, if people seek norm-relevant information only when transgression is met with negative consequences, we should not conclude that they intrinsically value such 
information. In this case, the underlying motive for obtaining information is simply to avoid punishment or loss of reputation.

Our findings also provide one possible explanation why there is inconsistency in norm compliance that is not correlated with the presence or absence of punishment, and how norm compliance might be context-dependent ${ }^{8}$. In those cases in which people are not willing to incur costs to obtain norm-revealing information, whether information affects decision-making depends on the diffusion, transparency and accessibility of the information. In cases where norm-relevant information is freely available, people's behavior will be influenced by the observation of what others do or by knowing what others expect them to do. In contrast, when norm-relevant information is less transparent and costly to obtain, there should be more variability of individual behavior and more selfish choices, as individuals will not make an effort to find out information about what other people do or believe ought to be done.

Our findings have implications for policies designed to promote pro-social norms, or change inefficient ones, especially when it is hard (or unacceptable) to punish transgressions. In particular, in cases where information regarding norm conformity is relatively private (e.g., the efforts individuals make to save energy) we might expect that people will not incur a cost to find out what others would do or think should be done. Consequently, deliberate efforts at promoting and diffusing norm-related information could be both important and valuable.

\footnotetext{
${ }^{8}$ As we pointed out above, our findings are based on a dictator game, which may be different from other environments in which there are negative consequences of norm violation, such as punishment or negative reputation effects. For example, unlike the dictator game situation, in an ultimatum game, what the responder thinks is fair can affect the proposer's payoff. In such situations agents are clearly motivated to obtain norm-relevant information and we expect to observe more widespread norm compliance.
} 


\section{References}

Bardsley, N., \& Sausgruber, R. (2005). "Conformity and reciprocity in public good provision”. Journal of Economic Psychology, 26(5), 664-681.

Bardsley, N. (2007). “Dictator game giving: altruism or artefact?” Experimental Economics, DOI 10.1007/s10683-007-9172-2.

Berg J., Dickhaut, J. and McCabe K (1995) “Trust, Reciprocity and Social History.” Games Economic Behavior 10, 122-142.

Bicchieri, C. (2006). The Grammar of Society: the Nature and Dynamics of Social Norms, New York: Cambridge University Press.

Bicchieri, C. and Xiao, E. (2008). "Do the right thing: But only if others do so”. Journal of Behavioral Decision Making, 21, 1-18.

Bichieri, C. and Chavez, A. (2009). "Behaving as Expected: Public Information and Fairness Norms". Journal of Behavioral Decision Making (in press)

Cason, T. N. \& Mui, V. (1998). “Social Influence in the Sequential Dictator Game”. Journal of Mathematical Psychology. 42, 248-265.

Cialdini, R.B., Kallgren, C. A., \& Reno R.R.(1990). “A Focus Theory of Normative Conduct: A Theoretical Refinement and Reevaluation of the Role of Norms in Human Behavior”. Advances in Experimental Social Psychology, 24, 201-234.

Cox, J. (2004). “How to identify trust and reciprocity”, Games and Economic Behavior 46, p260-281.

Dana, J., Weber, R. A. and Kuang, J., "Exploiting the moral wriggle room : Experiments demonstrating an illusory preference for fairness.” Economic Theory, 2007, 33(1): 67-80. 
Dawes CT, Fowler JH, Johnson T, McElreath R, Smirnov O. (2007) "Egalitarian Motives in Humans," Nature 446 (12), p 794-796

Deutsch, M. and H. B. Gerard (1955). "A study of normative and information social influences upon individual judgment." Journal of Abnormal and Social Psychology 51: 629-636.

Fehr, E. \& Urs, F. (2004). "Social norms and human cooperation” Trends in Cognitive Sciences, 8(4), 185-190.

Guala, F. \& Mittone, L. (2008). "Paradigmatic Experiments: the Dictator Game”. CEEL working paper.

Deutsch, M. and Gerard, H.B. (1955). “A Study of Normative and Information Social Influences Upon Individual Judgment”. Journal of Abnormal and Social Psychology 51: 629-636.

Kelley, H.H. (1952). “Two Functions of Reference Groups”. In G.E. Swanson et al. (eds.), Readings in Social Psychology. New York: Holt, Rinehart and Winston.

Krupka, E. \& Weber, R. (2008). "The Focusing and Observational Effects of Social Norms”. Carnegie Mellon University. Working paper

Ledyard, J. (1995). "Public Goods: A Survey of Experimental Research”. In J. Hagel and A. Roth (eds.), The handbook of Experimental economics. Princeton: Princeton University Press.

Meier, S. and Frey, B. (2004). "Social Comparisons and Pro-social Behavior: Testing “Conditional Cooperation in a Field Experiment”. American Economic Review, 94(5), pp. 1717-1722.

Sugden, R. (1998). "Normative expectations: the simultaneous evolution of institutions 
and norms". In Ben-Ner, A. and Putterman, L. (Eds), Economics, Value, andOrganization. Cambridge: Cambridge University Press.

Sugden, R. (2004). The Economics of Rights, Cooperation and Welfare, second edition, Palgrave Macmillan, 154-165. 
Figure 1. Distribution of choices of viewing information in FI treatment $(\mathrm{N}=32)$

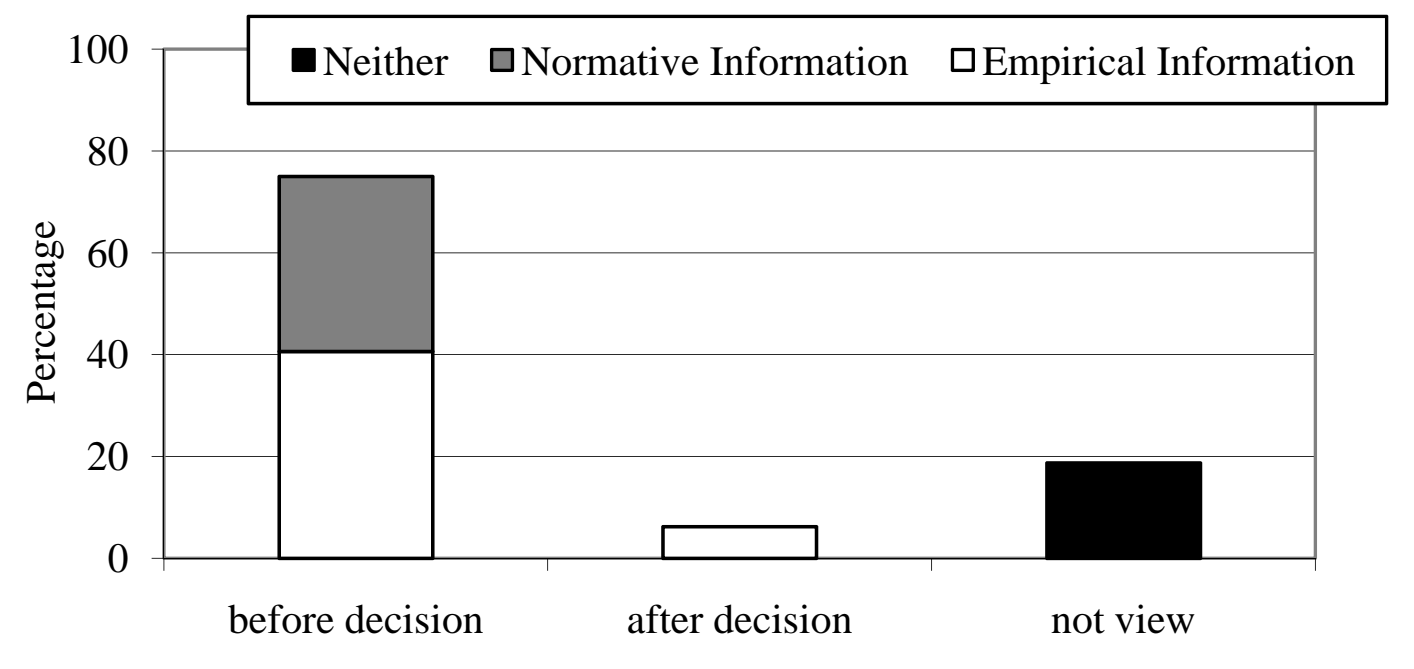

View information

Figure 2. Distribution of choices of buying information

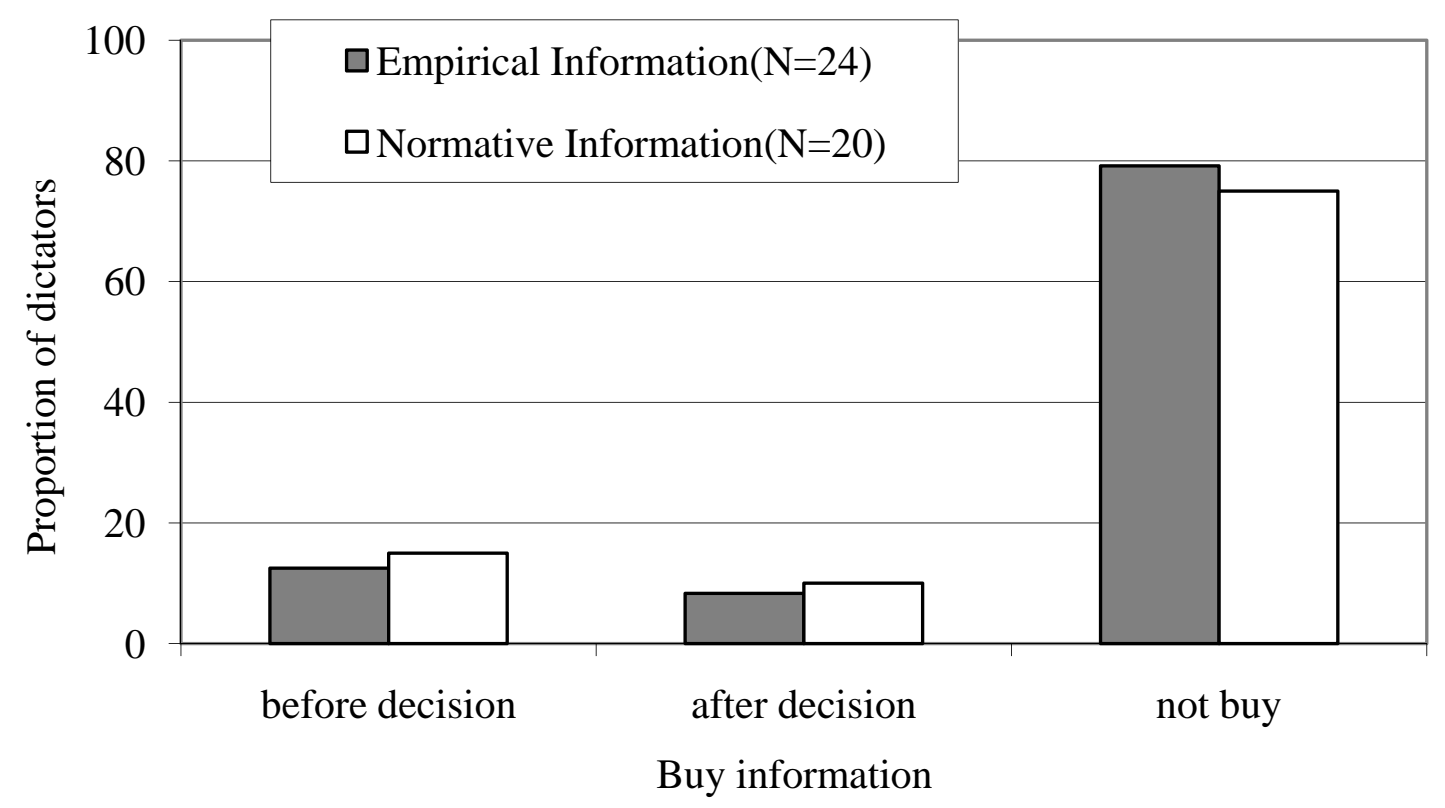


Table 1 Labels for the five envelopes (The order of the labels is randomized in the experiment)

Envelope Label

\section{Free Information Treatment}

I If you DO NOT want to view either (A) or (B), please open envelope I.

Envelope I contains only a decision card.

Your payment envelope that you will pick up at the end of the experiment will contain only your cash payment.

II If you would like to view (A) after your decision, please open envelope II.

Envelope II contains only a decision card.

Your payment envelope that you will pick up at the end of the experiment will contain your cash payment and a card with information (A).

III If you would like to view (B) after your decision, please open envelope III.

Envelope III contains only a decision card.

Your payment envelope that you will pick up at the end of the experiment will contain your cash payment and a card with information (B).

IV If you would like to view (A) before your decision, please open envelope IV.

Envelope IV contains a card with information (A) and a decision card.

Your payment envelope that you will pick up at the end of the experiment will contain only your cash payment.

$\mathrm{V} \quad$ If you would like to view (B) before your decision, please open envelope $\mathrm{V}$. Envelope $\mathbf{V}$ contains a card with information (B) and a decision card.

Your payment envelope that you will pick up at the end of the experiment will contain only your cash payment.

\section{Buy Information Treatments}

I If you would like view the information before your decision, please open envelope I. \$0.15 will be deducted from your final earnings.

Envelope I contains a card with the information and a decision card.

Your payment envelope that you will pick up at the end of the experiment will contain only your cash payment.

II If you would like to view the information after your decision, please open envelope II. \$0.05 will be deducted from your final earnings.

Envelope II contains only a decision card.

Your payment envelope that you will pick up at the end of the experiment will contain your cash payment and a card with the information.

III If you DO NOT want to view the information, please open envelope III.

Envelope III contains only a decision card.

Your payment envelope that you will pick up at the end of the experiment will contain only your cash payment. 
Table 2. Messages

\section{Message}

Fair normative information

$60 \%$ of the dividers who participated in a session of this experiment last year said that dividers should share the amount approximately equally (i.e., choose option C or D (their counterpart gets $40 \%$ or more)).

Selfish normative information

$60 \%$ of the dividers who participated in a session of this experiment last year said that dividers should approximately maximize their own earnings (i.e., choose option A or B (their counterpart gets $20 \%$ or less)).

Fair empirical information

$60 \%$ of the dividers who participated in a session of this experiment last year shared the amount approximately equally (i.e., chose option C or D (their counterpart got $40 \%$ or more)).

Selfish empirical information

$60 \%$ of the dividers who participated in a session of this experiment last year approximately maximized their own earnings (i.e., chose option A or B (their counterpart got 20\% or less)).

Note: The data reported in the messages were drawn from different sessions of the previous experiments. It was made clear to dictators that the information is from one session of the experiment. 


\section{Appendix A}

\section{Dictator's instruction for the dictator game}

\section{Instructions}

Thank you for coming! You've earned $\$ 5$ for showing up on time. Whatever you earn in the rest of the session will be in addition to this $\$ 5$. The instructions explain how you can make decisions. Please read these instructions carefully! There is no talking at any time during this experiment. If you have a question please raise your hand, and an experimenter will assist you.

You will be randomly and anonymously paired with another person in this room. You will never be informed of the identity of this person, either during or after the experiment. Similarly, your matched participant will never be informed about your identity. You are in the role of Divider and your matched participant will be referred to as your Counterpart. You and your Counterpart will participate only once in this decision problem. All the decisions will be anonymous.

This is how the experiment works.

Your task is to divide $\$ 10$ between the two of you. How much money you end up with at the end of the experiment depends on the decisions you make.

\section{Divider (You)}

You will choose a Dividing Option (described in detail below). A dividing Option determines how much of $\$ 10$ will go to the Divider (you) and how much will go to your Counterpart.

\section{Dividing Option}

The possible divisions appear in the table below. You must choose only one of them.

\begin{tabular}{|c|l|}
\hline Possible Dividing Options & The option is \\
\hline A & Divider gets $\mathbf{\$ 9}$ and Counterpart gets $\mathbf{\$ 1}$ \\
\hline B & Divider gets $\mathbf{8}$ and Counterpart gets $\mathbf{2}$ \\
\hline C & Divider gets $\mathbf{\mathbf { 6 }}$ and Counterpart gets $\mathbf{4}$ \\
\hline D & Divider gets $\mathbf{5}$ and Counterpart gets $\mathbf{5}$ \\
\hline E & Divider gets $\mathbf{4}$ and Counterpart gets $\mathbf{6}$ \\
\hline F & Divider gets $\mathbf{\$ 2}$ and Counterpart gets $\mathbf{8}$ \\
\hline G & Divider gets $\mathbf{1}$ and Counterpart gets $\mathbf{9}$ \\
\hline
\end{tabular}

\section{Experiment Procedure:}

\section{Step 1: Random and anonymous assignment of counterparts}

Each of you has randomly chosen a manila envelope. Your ID letter is shown on the last page of the instructions. Persons in this room who get the same ID will be paired. Please do not show anyone your ID letter. 


\section{Step 2: Divider chooses the option}

The Divider will be given a card where s/he can write down her/his decision and her/his ID. Below is a sample decision card:

Divider: (Dividing option)

I choose dividing option
ID: . That is,

Divider gets $\$$ Counterpart gets $\$$

After making the decision, the Divider will put the decision card into his/her manila envelope. After each Divider has finished, an experimenter will collect all the manila envelopes.

\section{Step 3: The Counterpart receives the Divider's decision.}

The experimenter will give each Divider's decision card to his/her Counterpart according to the ID on the card. The Counterpart will see the decision made by the Divider. After each Counterpart has finished an experimenter will collect all the cards.

\section{Step 4: Receive cash payment privately}

To keep everyone's decision and earnings anonymous, the experimenter will put each participant's earnings in an envelope marked with her/his ID letter. All Dividers' envelopes will be placed on one desk, and all Counterparts' envelopes will be placed on a different desk. Then, Dividers will be called one by one. When called, each Divider will pick up the envelope labeled with her/his letter ID. Then the Divider will exit the lab and drop all other supplies into the box outside the lab door. Every Counterpart will be paid in the same way after all the Dividers have been paid and have left the lab.

The Divider and Counterpart will remain anonymously matched at all times during the experiment. Even the experimenter will not know your decisions.

\section{End of Instructions}

\section{Your ID:}




\section{Dictator's instructions for envelopes}

You can open ONLY one of the attached five envelopes. Please read the following instructions carefully.

This experiment has been conducted before. The following information and instructions are available to the dividers in today's experiment:

(In Free Information treatment)

Information: (A and B are not based on behavior in the same session)

(A) The offers chosen by $60 \%$ of dividers in a previous session of this experiment.

(B) The offers that $60 \%$ of dividers in a previous session of this experiment said dividers should make.

You can view A or B (but not both) before or after your decision, or you can choose not to view either.

Your available choices (please pick one):

I. If you DO NOT want to view either (A) or (B), please open envelope I. Envelope I contains only a decision card.

Your payment envelope that you will pick up at the end of the experiment will contain only your cash payment.

II. If you would like to view (A) after your decision, please open envelope II.

Envelope II contains only a decision card.

Your payment envelope that you will pick up at the end of the experiment will contain your cash payment and a card with information (A).

III. If you would like to view (B) after your decision, please open envelope III.

Envelope III contains only a decision card.

Your payment envelope that you will pick up at the end of the experiment will contain your cash payment and a card with information (B).

IV. If you would like to view (A) before your decision, please open envelope IV.

Envelope IV contains a card with information (A) and a decision card.

Your payment envelope that you will pick up at the end of the experiment will contain only your cash payment.

V. If you would like to view (B) before your decision, please open envelope $V$.

Envelope $\mathbf{V}$ contains a card with information (B) and a decision card.

Your payment envelope that you will pick up at the end of the experiment will contain only your cash payment.

Now please open your chosen envelope and fill in the decision card. After you finish, please put all the five envelops and the decision card back into the big manila envelope and hand it to the experimenter. Only your decision card will be delivered to your Counterpart.

(In Buy Empirical Information treatment)

Information: The offers chosen by $60 \%$ of dividers in a previous session of this experiment.

(In Buy Normative Information treatment)

Information: The offers that $60 \%$ of dividers in a previous session of this experiment said dividers should make.

(The following instructions are the same for Buy Empirical Information and Buy Normative Information treatments) 
You can pay $\$ 0.15$ to view the information before your decision, or pay $\$ 0.05$ to view the information after your decision, or you can choose not to view it.

Your available choices (please pick one):

I. If you would like view the information before your decision, please open envelope I. $\quad \mathbf{\$ 0 . 1 5}$ will be deducted from your final earnings.

Envelope I contains a card with the information and a decision card.

Your payment envelope that you will pick up at the end of the experiment will contain only your cash payment.

II. If you would like to view the information after your decision, please open envelope II. \$0.05 will be deducted from your final earnings.

Envelope II contains only a decision card.

Your payment envelope that you will pick up at the end of the experiment will contain your cash payment and a card with the information.

III. If you DO NOT want to view the information, please open envelope III.

Envelope III contains only a decision card.

Your payment envelope that you will pick up at the end of the experiment will contain only your cash payment.

Now please open your chosen envelope and fill in the decision card. After you finish, please put all the three envelops and the decision card back into the big manila envelope and hand it to the experimenter.

Only your decision card will be delivered to your Counterpart. 